\title{
Understanding neutrino properties in a 2HDM see-saw
}

\author{
Alejandro Ibarra and Cristoforo Simonetto* \\ Technical University - Munich \\ E-mail: Alejandro. Ibarra@ph.tum.de, Cristoforo.Simonetto@ph.tum.de
}

We consider an extension of the Standard Model by heavy right-handed neutrinos and heavy Higgs doublets and show, using a renormalization group approach, that this model can simultaneously provide an explanation for the small neutrino masses and for the mild hierarchy observed between the atmospheric and the solar mass splittings, even when the extra degrees of freedom are very heavy. We analyse the necessary conditions to reproduce the oscillation parameters and we discuss possible experimental signatures of this model.

The 2011 Europhysics Conference on High Energy Physics-HEP 2011,

July 21-27, 2011

Grenoble, Rhône-Alpes France

${ }^{*}$ Speaker. 


\section{Introduction}

Extending the particle content of the SM with heavy right-handed neutrinos, thus implementing the (type I) see-saw mechanism, solves very elegantly the problem of small neutrino masses. Unfortunately, the see-saw mechanism tends to predict a neutrino mass hierarchy which is much larger than the one inferred from experiments [1]. Namely, under the plausible assumption that the neutrino Yukawa couplings have hierarchical eigenvalues, as observed in the quark and the charged lepton sectors, the mild mass hierarchy observed between the solar and the atmospheric mass splitting can only be accommodated in very special cases. One possibility arises when the hierarchy between the masses of the heavy right-handed neutrinos is much larger than the hierarchy between the neutrino Yukawa eigenvalues, in which case the right-handed mixing angles have to be tiny. Alternatively, when the hierarchy in the masses of the heavy right-handed neutrinos is comparable to the hierarchy in the Yukawa couplings, it is possible to accommodate the observed mild neutrino mass hierarchy only for certain, possibly fine-tuned, choices of the right-handed mixing angles.

On the other hand, in the general 2HDM, the see-saw can naturally lead to a mild neutrino mass hierarchy, via a combination of tree-level and loop induced neutrino mass [2]. Here we analyse this mechanism in a RG approach, following our publication [3]. It will turn out the mass hierarchy to depend only mildly on the masses of the Higgs fields. Therefore, by sufficient decoupling of the second Higgs doublet, the observed neutrino mass hierarchy can be explained while the successes of the SM are preserved. Finally we will discuss the radiative corrections to the leptonic mixing angles and discuss a possible signature.

\section{Neutrino masses in a 2HDM extended with right-handed neutrinos}

In the general 2HDM extended with right-handed neutrinos, the part of the Lagrangian involving right-handed neutrinos is given by:

$$
-\mathscr{L}^{v}=\left(Y_{v}^{a}\right)_{i j} \bar{l}_{L i} v_{R j} \tilde{\Phi}_{a}-\frac{1}{2} M_{i j} \bar{v}_{R i}^{C} v_{R j}+\text { h.c. }, \quad a=1,2 .
$$

We will assume that the mass scale of the right-handed neutrinos is much larger than $m_{Z}$ and the masses of the extra Higgs fields $H^{0}, A^{0}, H^{ \pm}$, which we denote collectively by $m_{H}$. Furthermore, we will simplify the notation by assuming without loss of generality $\left\langle\Phi_{2}\right\rangle=0$. Then the neutrino mass can be written at tree-level:

$$
\mathscr{M}_{v}^{\text {tree }}=\frac{v^{2}}{2} Y_{v}^{1} M^{-1}\left(Y_{v}^{1}\right)^{T}
$$

Moreover we will concentrate on a see-saw model with just one right-handed neutrino with mass $M$. Embedded in a more realistic model with three right-handed neutrinos it corresponds to the one giving the largest contribution to the left-handed neutrino mass. As discussed in the introduction, generically the other contributions are expected to be small. Then, with this assumption, the neutrino Yukawa couplings, $Y_{v}^{a}$, are 3-vectors and at tree-level only one neutrino obtains a mass:

$$
m_{3}^{\text {tree }}=\frac{v^{2}}{2 M}\left|Y_{v}^{1}\right|^{2}
$$


However, in order to compare the predictions of the model with low energy experiments one must take into account radiative corrections. Therefore we will make use of the $\beta$-functions [4] to evolve the Weinberg operator to the scale of the second Higgs doublet $m_{H}$. Even if $m_{H} \gg m_{Z}$ the running below $m_{H}$ will only modify the values of the neutrino mass by a small factor [5].

We find, in the leading-log approximation:

$$
m_{2}=\frac{1}{16 \pi^{2}} \frac{\left|\lambda_{5}\right| v^{2}}{M}\left[\left|Y_{v}^{2}\right|^{2}-\frac{\left|Y_{v}^{2 \dagger} Y_{v}^{1}\right|^{2}}{\left|Y_{v}^{1}\right|^{2}}\right] \log \frac{M}{m_{H}},
$$

which depends linearly on the quartic coupling $\frac{1}{2} \lambda_{5}\left(\Phi_{1}^{\dagger} \Phi_{2}\right)^{2}$.

It is interesting to note that, under some well motivated assumptions, the hierarchy between the tree level mass $m_{3}$ and the radiatively generated neutrino mass $m_{2}$ can be fairly mild. For instance, taking the typical values $\left|\lambda_{5}\right| \sim 1, \log \left(M / m_{H}\right) \sim 30$ and assuming Yukawa couplings of comparable strengths $\left|Y_{v}^{2}\right| \sim\left|Y_{v}^{1}\right|$, which are neither aligned nor orthogonal to each other, one obtains a mild mass hierarchy, in qualitative agreement with the experimental data:

$$
m_{2} / m_{3} \sim 0.2 .
$$

Note that, whereas the overall scale of the light neutrino masses depends linearly on the inverse of $M$, the ratio between the two heaviest neutrino mass eigenvalues depends only logarithmically on the masses of the new particles. Therefore the problems usually associated with the general 2HDM, as the possibility of breaking of electric charge and generically large contributions to electroweak precision observables, FCNCs and LFV in conflict with experiment, can be evaded in the decoupling limit, where one Higgs doublet is rather heavy.

\section{Corrections to the mixing angles}

The leptonic mixing matrix might receive radiative corrections from many contributions. Analogously to the radiative generation of $m_{2}$, some of them are naturally as large as $\sim 0.2$. Therefore, predictions from flavour symmetries can be perturbed by rather large corrections.

For example assume the phenomenologically motivated case of maximal atmospheric mixing and vanishing $\theta_{13}$ at some high scale. Even in this extreme scenario the radiative corrections can easily drive the mixing angles to values which lie outside the experimentally allowed region. Moreover, it is interesting that if the charged lepton Yukawa couplings are sub-dominant in the corrections to the leptonic mixing matrix, then a correlation arises between the deviations of $U_{23} / U_{33}$ and $U_{13}$ from their corresponding values at the cut-off scale. Concretely, in the case when at the cut-off scale the atmospheric mixing angle is exactly maximal and $\theta_{13}$ vanishes, at low energies the elements of the leptonic mixing matrix approximately satisfy

$$
\theta_{23}-\frac{\pi}{4} \simeq \sqrt{2} \sin \theta_{13} \cos \delta
$$

If there are additional sources of lepton flavour violation, then the low energy values of $\theta_{23}$ and $\theta_{13}$ are expected to deviate from this relation. This is illustrated in the scatter plot shown in fig. 1, which has been obtained by the numerical one loop integration of the RGEs of the 2HDM extended by one right-handed neutrino. The correlation, eq. (3.1) is shown as a black solid line. To investigate the impact of the charged lepton Yukawa couplings we have examined several cases shown in different colours. For more details see [3]. 


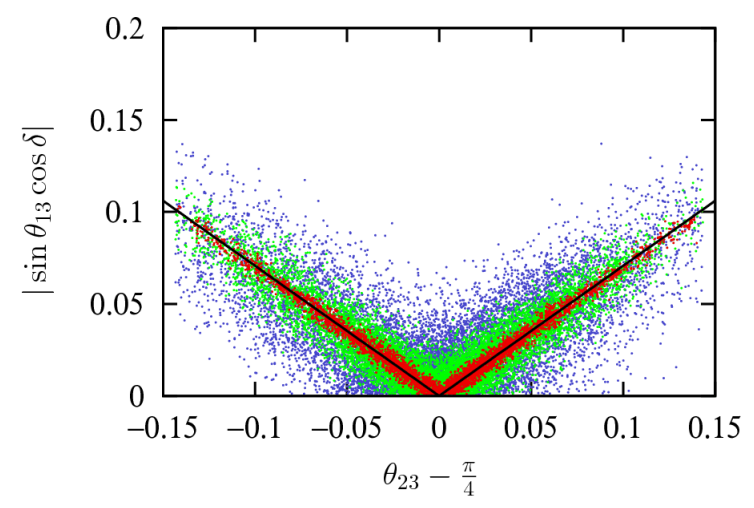

Figure 1: Scatter plot showing $\left|\sin \theta_{13} \cos \delta\right|$ against $\theta_{23}-\frac{\pi}{4}$ at low energies for the case of tri-bi-maximal mixing at the Majorana mass scale and random choices of high energy parameters consistent with the measured neutrino oscillation parameters.

\section{Conclusions}

In the see-saw in the general 2HDM, the mild hierarchy between the two heaviest neutrino masses can be obtained naturally, even with hierarchical neutrino Yukawa eigenvalues. Since the mass hierarchy depends only logarithmically on the mass of the extra Higgs doublet, the phenomenological constraints on the general 2HDM can be largely alleviated by decoupling.

Analogously to the radiative generation of the next-to-heaviest neutrino mass, a non-vanishing value for $\theta_{13}$ is induced by the RG evolution even if it vanishes at high scales. Therefore, we expect hierarchies in the mixing angles not to be strong. If the leptonic mixing matrix was exactly tri-bi-maximal at the Majorana mass scale, a correlation may arise between $\theta_{23}$ and $\theta_{13}$.

\section{Acknowledgments}

This work was partially supported by the DFG cluster of excellence Origin and Structure of the Universe and by the Graduiertenkolleg "Particle Physics at the Energy Frontier of New Phenomena".

\section{References}

[1] J. A. Casas, A. Ibarra and F. Jimenez-Alburquerque, Hints on the high-energy seesaw mechanism from the low-energy neutrino spectrum, JHEP 0704 (2007) 064, [hep-ph/ 061228 ].

[2] W. Grimus, H. Neufeld, Three neutrino mass spectrum from combining seesaw and radiative neutrino mass mechanisms, Phys. Lett. B486 (2000) 385-390, [hep-ph/9911465].

[3] A. Ibarra and C. Simonetto, Understanding neutrino properties from decoupling right-handed neutrinos and extra Higgs doublets, JHEP 1111 (2011) 022 [arXiv: 1107.2386 [hep-ph] ].

[4] W. Grimus, L. Lavoura, Renormalization of the neutrino mass operators in the multi-Higgs-doublet standard model, Eur. Phys. J. C39 (2005) 219-227, [hep-ph/ 0409231$].$

[5] J. A. Casas, J. R. Espinosa, A. Ibarra, I. Navarro, General RG equations for physical neutrino parameters and their phenomenological implications, Nucl. Phys. B573 (2000) 652-684, [hep-ph/9910420]. 